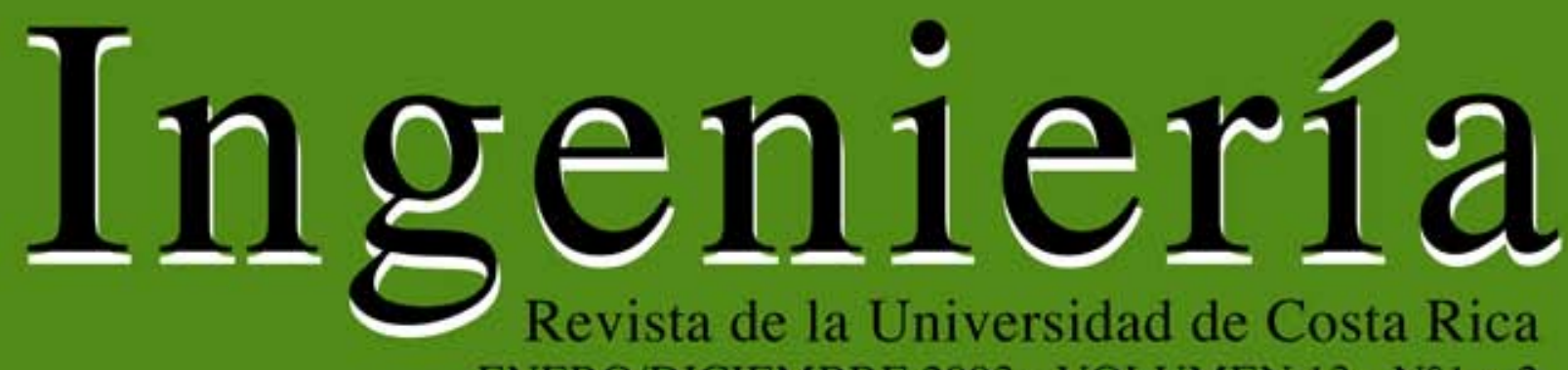
ENERO/DICIEMBRE 2003 - VOLUMEN 13 - N¹ y 2

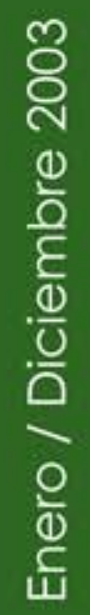

$\frac{m}{5}$
$\frac{c}{d}$
$\frac{5}{5}$
$\frac{\partial}{\circ}$

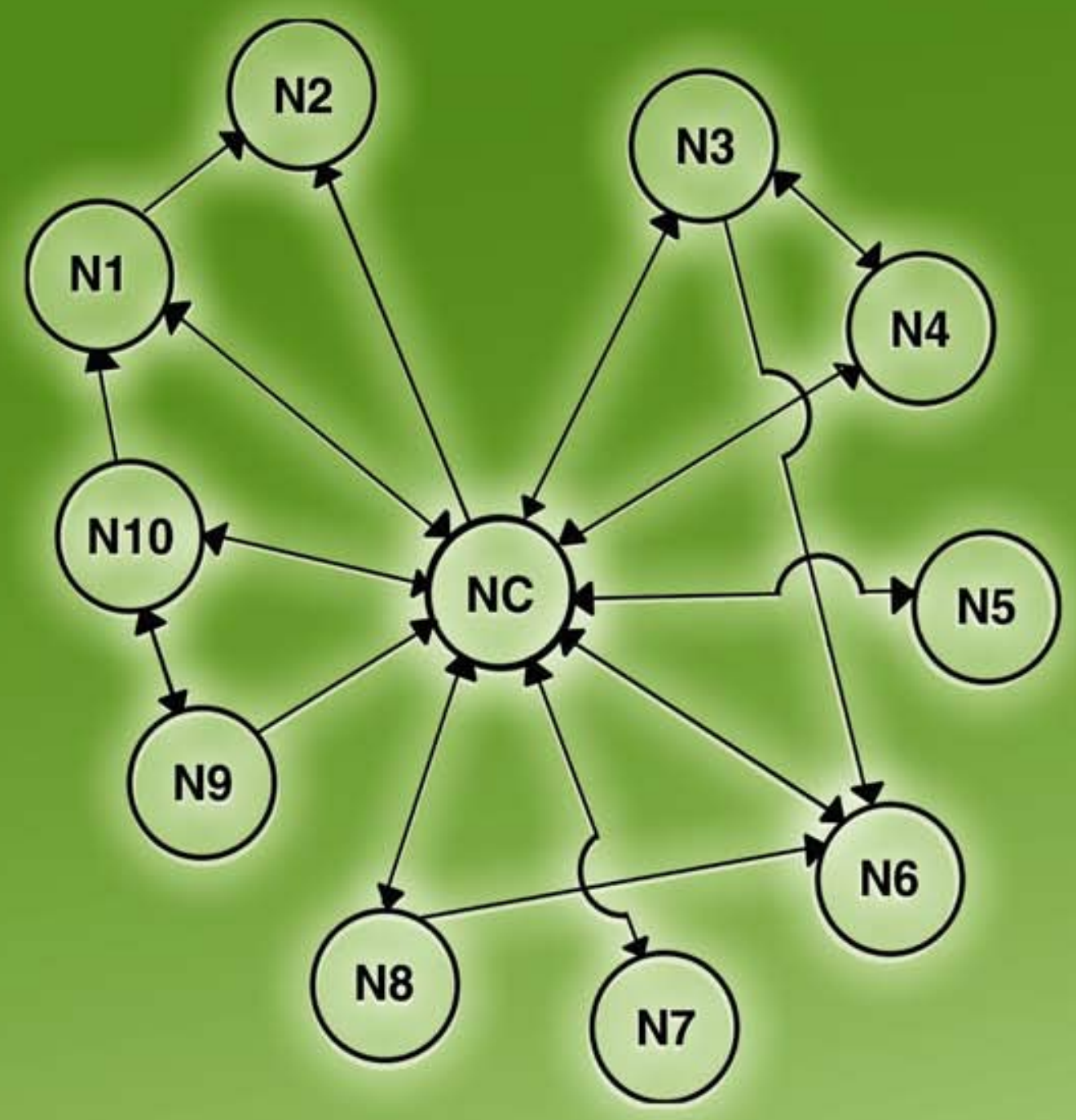

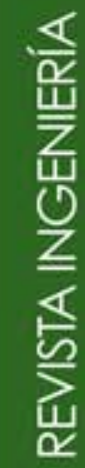




\title{
NUEVAS APROXIMACIONES DEL TIEMPO MUERTO PARA ESTUDIOS DE CONTROL
}

Víctor M. Alfaro Ruiz

\begin{abstract}
Resumen
Se enfrenta el problema que origina el tiempo muerto de la planta, en el estudio del comportamiento de los sistemas de control, realizando una comparación entre diferentes aproximaciones presentadas para éste en la literatura técnica. Se determinó que la aproximación de segundo orden de Sthal y Hippe, es superior a las de Padé y a otras de las existentes.

Se presentan nuevas ecuaciones para aproximar el tiempo muerto de una planta y se muestra que las mismas proveen mejores resultados que las ecuaciones ya propuestas.
\end{abstract}

Palabras clave: control automático, retardos de tiempo, aproximaciones.

\begin{abstract}
The problem related to the time delay approximations in Control Systems Theory is addressed by making a comparison among different approximations presented in the technical literature.

It is determined that the second order approximation proposed by Sthal and Hippe surpasses the Padé approximation and other existing approximations.

New equations to approximate the system time delay are presented and it is shown that they give better results than the equations so far proposed.
\end{abstract}

Keywords: automatic control, time delay, approximations.

\section{INTRODUCCIÓN}

El estudio analítico o por simulación de los sistemas de control realimentado, involucra normalmente la manipulación de las funciones de transferencia de los dispositivos que lo componen, como lo son el controlador, el proceso controlado y los instrumentos de medición y actuación.

Suponiendo que el lazo de control realimentado es el mostrado en la Figura 1, en donde $G_{C}(s)$ es la función de transferencia del controlador y $G_{p}(s)$ la del conjunto actuador sensor, a la que se llamará simplemente planta, la función de transferencia de lazo cerrado es:
$\frac{y(s)}{r(s)}=M(s)=\frac{G_{C}(s) G_{p}(s)}{1+G_{C}(s) G_{p}(s)}$

Si la planta tiene tiempo muerto, sea este real o producto del modelado del sistema, su función de transferencia se puede escribir como

$$
G_{p}(s)=G_{p}^{\prime}(s) e^{-t_{m} s}
$$

Sustituyendo (2) en (1) se tiene que $M(s)$ está dada por

$M(s)=\frac{G_{C}(s) G_{p}^{\prime}(s) e^{-t_{m} s}}{1+G_{C}(s) G_{p}^{\prime}(s) e^{-t_{m} s}}$ 


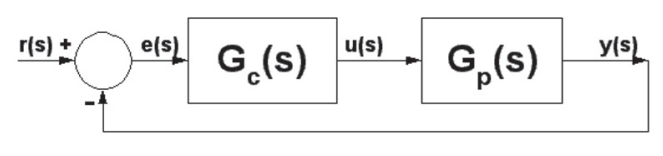

Figura 1. Sistema de control realimentado.

El término exponencial en el numerador no representa ningún problema para el análisis; corresponde al tiempo muerto de la planta que aparece en la respuesta del sistema de lazo cerrado. Sin embargo, el polinomio característico del sistema de lazo cerrado es

$$
p(s)=1+G_{C}(s) G_{p}^{\prime}(s) e^{-t_{m} s}
$$

el cual no es una función racional en $s$.

Para poder realizar estudios del desempeño, de la estabilidad y el diseño del sistema de control, es necesario aproximar entonces el tiempo muerto por alguna función racional en $s$, usualmente mediante un cociente de polinomios.

Se ha encontrado en la literatura de control la utilización de diversas aproximaciones del tiempo muerto, algunas propuestas como mejores sin justificación, razón por lo que se deseó realizar pruebas comparativas de varias de estas aproximaciones y estudiar la posibilidad de establecer nuevas expresiones con un mejor desempeño. Los resultados de este estudio se detallan adelante.

\section{APROXIMACIONES DEL TIEMPO MUERTO}

Se definirá primero la función tiempo muerto como

$f_{0}(s)=e^{-t_{m} s}$

y se establecerán las aproximaciones de ésta que se desean comparar.

\subsection{Mediante series de Taylor}

La función $f_{O}(s)$ puede expandirse en una serie de Taylor como (Dwight, 1961)

$e^{-t_{m} s} \approx 1-t_{m} s+\frac{t_{m}^{2} s^{2}}{2 !}-\frac{t_{m}^{3} s^{3}}{3 !}+\ldots$

de donde la aproximación mediante una serie de primer orden sería

$e^{-t_{m} s} \approx f_{1}(s)=1-t_{m} s$

y mediante una de segundo orden

$e^{-t_{m} s} \approx f_{2}(s)=1-t_{m} s+\frac{t_{m}^{2} s^{2}}{2 !}$

(Martin et al, 1975) utilizaron la aproximación $f_{l}(s)$ en su procedimíento de síntesis de controladores.

También se puede aproximar el tiempo muerto por el cociente de dos series de Taylor de la forma

$e^{-t_{m} s} \approx \frac{e^{-\frac{t_{m}}{2} s}}{e^{\frac{t_{m}}{2} s}}=\frac{1-\left(\frac{t_{m}}{2}\right) s+\frac{\left(\frac{t_{m}}{2}\right)^{2} s^{2}}{2 !}-\frac{\left(\frac{t_{m}}{2}\right)^{3} s^{3}}{3 !}+\ldots}{1+\left(\frac{t_{m}}{2}\right) s+\frac{\left(\frac{t_{m}}{2}\right)^{2} s^{2}}{2 !}+\frac{\left(\frac{t_{m}}{2}\right)^{3} s^{3}}{3 !}+\ldots}$

La aproximación de primer orden sería entonces

$e^{-t_{m} s} \approx f_{3}(s)=\frac{1-\left(t_{m} / 2\right) s}{1+\left(t_{m} / 2\right) s}=\frac{2-t_{m} s}{2+t_{m} s}$

y la de segundo orden

$e^{-t_{m} s} \approx f_{4}(s)=\frac{1-t_{m} / 2 s+t_{m}^{2} / 8 s}{1+t_{m} / 2 s+t_{m}^{2} / 8 s}=\frac{8-4 t_{m} s+t_{m}^{2} s^{2}}{8+4 t_{m} s+t_{m}^{2} s^{2}}$

\subsection{Aproximaciones de Padé}

Las aproximaciones de Padé para el tiempo muerto, son de las más populares en los estudios de control por las características que este mismo estudio permitió confirmar. 
Por ejemplo (Yuwana y Seborg, 1982) utilizaron una aproximación de Padé de primer orden como parte de su procedimiento de identificación, (Rivera et al, 1986) utilizaron aproximaciones de Padé de orden cero y de primer orden en la derivación de sus fórmulas IMC para sintonización de controladores.

La expresión general de las aproximaciones de Padé es

$$
e^{-t_{m} s}=\frac{1+\sum_{i=1}^{n} \frac{i !\left(-t_{m} s\right)^{i}}{(2 i) !}}{1+\sum_{i=1}^{n} \frac{i !\left(t_{m} s\right)^{i}}{(2 i) !}}
$$

Para el caso $n=1$ (Padé de primer orden) se tiene

$e^{-t_{m} s} \approx \frac{1-\left(t_{m} / 2\right) s}{1+\left(t_{m} / 2\right) s}=\frac{2-t_{m}}{2+t_{m}}$

que es igual a la aproximación $f_{3}(s)$ anterior y la aproximación de Padé de segundo orden

$$
e^{-t_{m} s} \approx f_{5}(s)=\frac{1-t_{m} / 2 s+t_{m}^{2} / 12 s}{1+t_{m} / 2 s+t_{m}^{2} / 12 s}=\frac{12-6 t_{m}+t_{m}^{2} s^{2}}{12+6 t_{m}+t_{m}^{2} s^{2}}
$$

\subsection{Mediante polos y ceros múltiples}

La función tiempo muerto se puede definir también como la respuesta de un número infinito de sistemas de primer orden en serie de la forma

$$
e^{-t_{m} s}=\lim _{n \rightarrow \infty} \frac{1}{\left(1+\frac{t_{m}}{n} s\right)^{n}}=\lim _{n \rightarrow \infty} \frac{\left(1-\frac{t_{m}}{2 n} s\right)^{n}}{\left(1+\frac{t_{m}}{2 n} s\right)^{n}}
$$

entonces, la aproximación de primer orden sería

$e^{-t_{m} s} \approx \frac{1-\left(t_{m} / 2\right) s}{1+\left(t_{m} / 2\right) s}=\frac{2-t_{m}}{2+t_{m}}$

que resulta ser igual a $f_{3}(s)$ y la de segundo

$$
e^{-t_{m} s} \approx f_{6}(s)=\frac{\left(1-\frac{t_{m}}{4} s\right)^{2}}{\left(1+\frac{t_{m}}{4} s\right)^{2}}=\frac{16-8 t_{m} s+t_{m}^{2} s^{2}}{16+8 t_{m} s+t_{m}^{2} s^{2}}
$$

\subsection{Otras aproximaciones}

(Jutan y Rodríguez, 1984) usaron como parte de su procedimiento de identificación con control $\mathrm{P}$ la aproximación

$e^{-t_{m} s} \approx f_{7}(s)=\frac{1-0,6143 t_{m} s+0,1247 t_{m}^{2} s^{2}}{1+0,3866 t_{m} s}$

(Bogere y Özgen, 1989) con el mismo propósito emplearon la expresión

$e^{-t_{m} s} \approx f_{8}(s)=1-0,8647 t_{m} s+0,226 t_{m}^{2} s^{2}$

(O’Dwyer,1996) incluyó en su tesis doctoral algunas de las aproximaciones para el tiempo muerto anteriores y las siguientes, identificadas con el nombre de su autor

- (Marshall, 1979)

$e^{-t_{m} s} \approx f_{9}(s)=\frac{1-0,0625 t_{m}^{2} s^{2}}{1+0,0625 t_{m}^{2} s^{2}}=\frac{16-t_{m}^{2} s^{2}}{16+t_{m}^{2} s^{2}}$

- (Piche,1990) (producto)

$e^{-t_{m} s} \approx \frac{1-0,5 t_{m} s+0.125 t_{m}^{2} s^{2}}{1+0,5 t_{m} s+0.125 t_{m}^{2} s^{2}}$

que es igual a $f_{4}(s)$

- $\quad$ Piche (Laguerre)

$e^{-t_{m} s} \approx \frac{1-0,5 t_{m} s+0,0625 t_{m}^{2} s^{2}}{1+0,5 t_{m} s+0,0625 t_{m}^{2} s^{2}}$

que es igual a $f_{6}(s)$ 
- (Gradshteyn y Ryzhik, 1980)

$e^{-t_{m} s} \approx f_{10}(s)=\frac{1-0,5 t_{m} s+0,1013 t_{m}^{2} s^{2}}{1+0,5 t_{m} s+0,1013 t_{m}^{2} s^{2}}$

(Sthaly Hippe, 1987) optimizaron la aproximación del tiempo muerto para reproducir la respuesta de frecuencia sobre el ámbito de frecuencias mayor posible y determinaron funciones de transferencia de segundo hasta quinto orden. $\mathrm{Su}$ aproximación de grado dos está dada por la función de transferencia

$e^{-t_{m} s} \approx f_{11}(s)=\frac{1-0,49 t_{m} s+0,0954 t_{m}^{2} s^{2}}{1+0,49 t_{m} s+0,0954 t_{m}^{2} s^{2}}$

\section{COMPARACIÓN DE LAS APROXIMACIONES}

Con la finalidad de evaluar la bondad de las aproximaciones del tiempo muerto $f_{1}(s)$ a $f_{11}(s)$ anteriores, se realizaron pruebas comparativas incluyendo: aproximación de la función exponencial, respuesta de un tiempo muerto puro, respuesta de un sistema de primer orden más tiempo muerto, determinación de la ganancia en el límite de la estabilidad y respuesta de frecuencia.

\subsection{Aproximación de la función exponencial}

Definiendo $x=t_{m} s$ se investigó qué tan bien se aproxima a la función $e^{-x}$ en el intervalo $0 \leq x \leq 2,0$.

La evaluación de las aproximaciones se realizó calculando los índices de error (IEAei) y calidad (ICAei) de las aproximaciones definidos en el Apéndice, apartedo 6.

En la Figura 2 y el Cuadro 1 se muestran las respuestas de las diferentes funciones. Como se puede ver, las series de Taylor $\left(f_{1}, f_{2}\right)$ y la ecuación de Marshall $\left(f_{9}\right)$ fallan al aproximar la función exponencial, mientras que la aproximación de Padé de segundo orden $\left(f_{5}\right)$ provee los mejores resultados. La aproximación de Padé de primer orden $\left(f_{3}\right)$, si bien no es buena si se considera todo el ámbito estudiado, su desempeño es aceptable hasta valores de $x=1,0$.

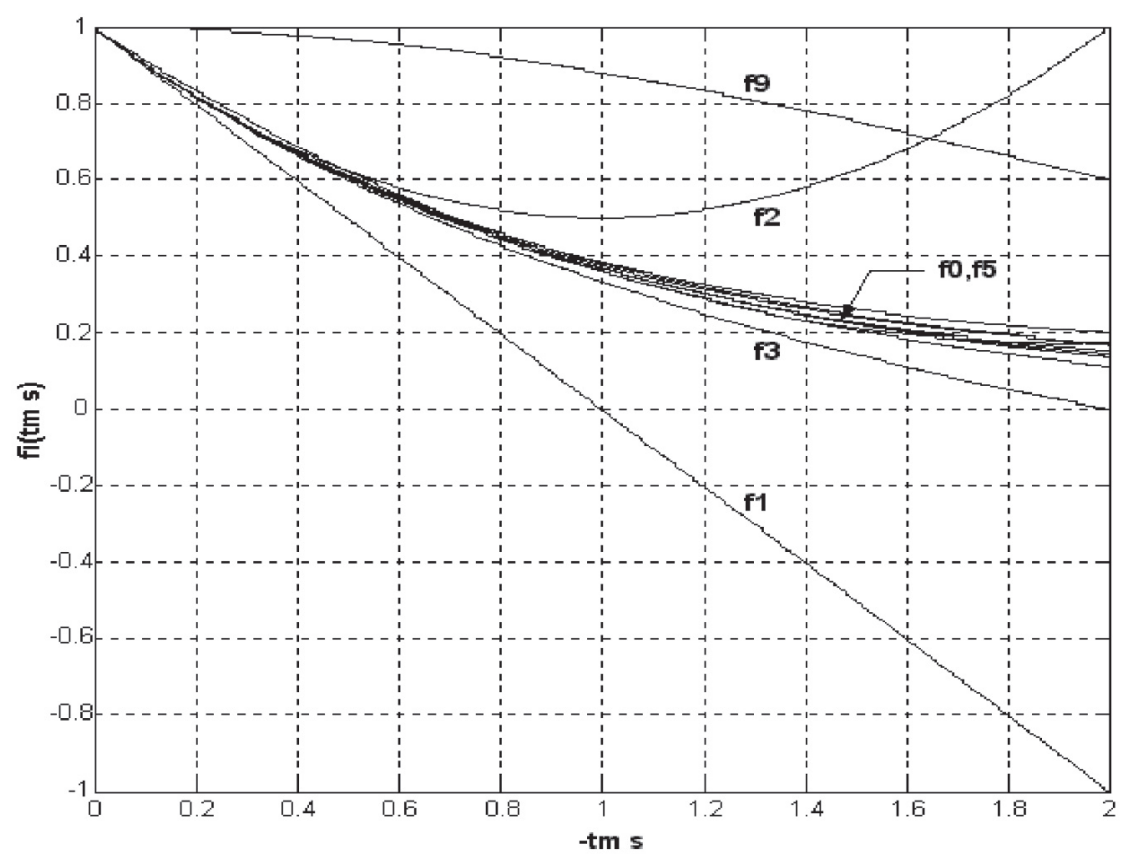

Figura 2. Aproximaciones de la función exponencial 
Cuadro 1. Aproximación de la función exponencial

\section{Función}

IEAei

ICAei

\begin{tabular}{lrr}
$f_{0}$ & \multicolumn{2}{c}{ ITMe $=0,8647(\mathrm{x}=[0,2])$} \\
$f_{1}$ & 0,8647 & 0 \\
$f_{2}$ & 0,46687 & 45,79 \\
$f_{3}$ & 0,0921 & 89,35 \\
$f_{4}$ & 0,0442 & 94,89 \\
$f_{5}$ & 0,0032 & 99,63 \\
$f_{6}$ & 0,0188 & 97,83 \\
$f_{7}$ & 0,0058 & 99,33 \\
$f_{8}$ & 0,0246 & 97,16 \\
$f_{9}$ & 0,8445 & 2,34 \\
$f_{10}$ & 0,0214 & 97,53 \\
$f_{11}$ & 0,0306 & 96,46
\end{tabular}

\subsection{Aproximación de un tiempo muerto puro}

Se consideraron para esta comparación, las aproximaciones que representan funciones de transferencia propias y se verificó, qué tan bien aproximaban estas un tiempo muerto puro, a partir de la respuesta a una entrada escalón unitario.

La comparación se realizó calculando los índices de error (IEAti) y calidad (ICAti) de las aproximaciones del tiempo muerto definidos en el Apéndice.

Las pruebas indicaron que un intervalo de tiempo igual a cuatro tiempos muertos $(m=4)$, era suficiente para asegurar que la respuesta de todas las funciones hubiera alcanzado su valor final.

La Figura 3 y el Cuadro 2 muestran los resultados obtenidos.

Las aproximaciones de segundo orden mostraron resultados muy similares entre sí, siendo la aproximación de Sthal y Hippe $\left(f_{11}\right)$ la mejor. La aproximación de Padé de primer orden $\left(f_{3}\right)$ fue la que mostró mayor error.

\subsection{Efecto en la respuesta al escalón}

Con frecuencia, las especificaciones del comportamiento deseado del sistema de control, se dan en función de la respuesta en el tiempo a una entrada del tipo escalón.

Los programas CACSD como MATLAB 6.5® o Scilab, no pueden manipular funciones de transferencia que incluyan tiempos muertos para obtener por ejemplo, la función de transferencia de lazo cerrado del sistema de control. Se debe utilizar una aproximación para los mismos, MATLAB $6.5 \circledR$ provee la función pade $(\mathrm{tm}, \mathrm{n})$ para esto, o emplear programas de simulación digital como Simulink 5.0® o VisSim 3.0 que proveen bloques operacionales para el tiempo muerto, simulado como una lista de datos almacenados en memoria, para realizar los estudios con este tipo de plantas.

Se investigó entonces el efecto de las aproximaciones en la respuesta de una planta de primer orden más tiempo muerto dada por

$G_{p}(s)=\frac{k_{p} e^{-t_{m} s}}{\tau s+1}$

En forma similar a como se hizo en las comparaciones anteriores, se calculó un índice de error (IEAyi) y uno de calidad (ICAyi) de las aproximaciones a la respuesta al escalón (ver Apéndice).

Se empleó en las pruebas una planta con ganancia unitaria $\left(k_{p}=1\right)$, constante de tiempo unitaria $(=1)$ y tiempo muerto variable ( $t m=0,10,250,5$ $0,751,0$ y 2,0$)$.

En la Figura 4 y en el Cuadro 3 se muestran los resultados de la simulación en función del tiempo muerto normalizado $o t_{m}$.

Como era de esperarse, todas las aproximaciones, incluso la de primer orden, dieron resultados aceptables cuando el tiempo muerto normalizado era bajo y éste empezó a deteriorarse a medida que o aumentó. 
Los mejores resultados se obtuvieron con la aproximación de Sthal y Hippe $\left(f_{11}\right)$ seguidos de la de Gradshteyn y Ryzhik $\left(f_{10}\right)$ y la de Padé de segundo orden $\left(f_{5}\right)$.

Si bien los resultados con la aproximación de Padé de primer orden muestran el menor índice de calidad, este no es significativamente menor que el obtenido con las aproximaciones de segundo orden.

\subsection{Efecto sobre la estabilidad del sistema de control}

Un aspecto muy importante en los estudios de los sistemas de control, es la determinación de las condiciones de estabilidad del mismo a partir de su polinomio característico, por lo que se investigó el efecto de las aproximaciones del tiempo muerto sobre ésta.

Se utilizó un sistema de control con un controlador proporcional $G_{C}(s)=K_{C}$ y una planta de primer orden más tiempo muerto, con los mismos parámetros que en la prueba anterior. Con éste se determinó el valor de la ganancia en el límite de la estabilidad $K_{\text {cuo }}$ con el tiempo muerto exacto, empleando el programa VisSim, y los valores de la ganancia límite con las aproximaciones $K_{c u i}$ resolviendo el polinomio característico $p_{i}\left(f_{i}(s), K_{c}, s\right)=0$ en forma iterativa empleando el programa Scilab.

Cuadro 2. Aproximación del tiempo muerto puro.

\section{Función}

IEAti

ICAti

$f_{0}$

$f_{3}$

$I T M t=3,\left(\mathrm{t}=\left[0,4 t_{m}\right], t_{m}=1\right)$

$f_{4}$

0,5770

80,77

$f_{5}$

0,4115

86,28

$f_{6}$

0,4057

86,48

$f^{6}$

0,4518

84,94

$f_{10}$

0,3954

86,82

$f_{11}$

0,3929

86,90

El Cuadro 4 muestra los valores de las ganancias en el límite de estabilidad obtenidas en función del tiempo muerto normalizado, así como los porcentajes de error de las mismas.

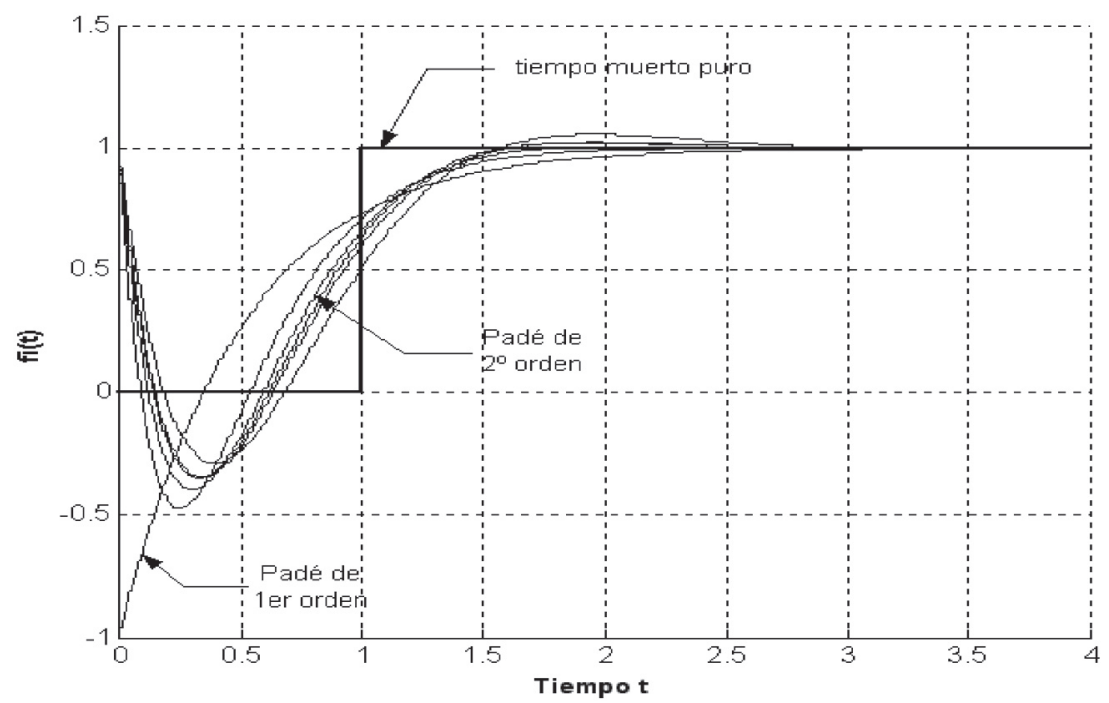

Figura 3. Respuesta a un escalón unitario, $\mathrm{tm}=1$ 
Como se aprecia en el Cuadro, cada aproximación mostró porcentajes de error similares al estimar la ganancia límite, con los diferentes tiempos muertos normalizados utilizados.

En esta prueba se hizo evidente que la aproximación de Padé de primer orden, no es adecuada para estudios de estabilidad ya que con ella se obtienen valores para la ganancia límite, aproximadamente un $30 \%$ mayores que el real.

La mejor estimación se obtuvo con la aproximación de Sthal y Hippe $\left(f_{11}\right)$, con un error

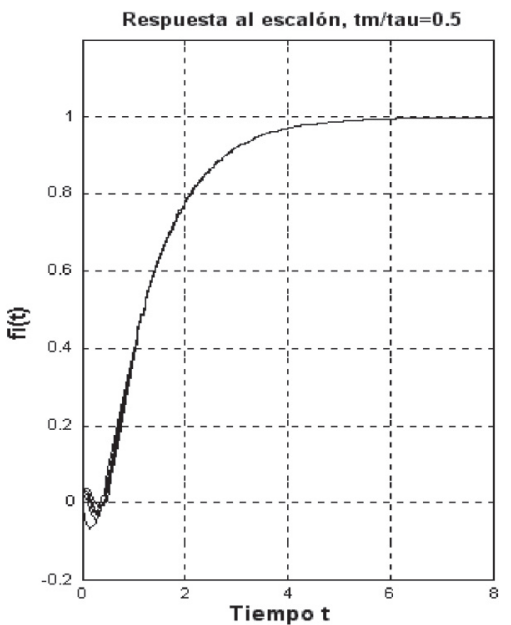

promedio de solo $-0,5 \%$, seguida de la de Padé de segundo orden $\left(f_{5}\right)$ con $+1,15 \%$ de error y de la de Gradshteyn y Ryzhik $\left(f_{10}\right)$ con $-2,3 \%$ de promedio la que, aunque con un error ligeramente mayor que el error de la aproximación de Padé, predice un valor conservador de la ganancia límite.

Es evidente el buen desempeño logrado con la aproximación de Sthal y Hippe ya que la ganancia predicha con ella, aparte de ser muy precisa, es segura por estar por debajo del valor real.

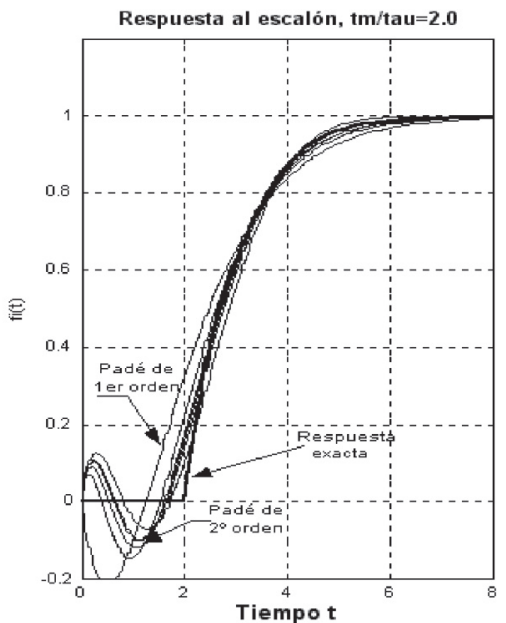

Figura 4. Respuesta del sistema de primer orden más tiempo muerto.

Cuadro 3. Efecto en la respuesta de un sistema de primer orden más tiempo muerto.

\begin{tabular}{|c|c|c|c|c|c|c|c|}
\hline \multicolumn{8}{|c|}{ ITMy } \\
\hline${ }_{o} t_{m}{ }^{\prime}$ & 0,10 & 0,25 & 0,50 & 0,75 & 1,0 & 1,5 & 2,0 \\
\hline$f_{0}$ & 8,90 & 8,75 & 8,50 & 8,25 & 8,00 & 7,50 & 7,00 \\
\hline \multicolumn{8}{|c|}{ IEAyi } \\
\hline$f_{3}$ & $1,70 \mathrm{e}-3$ & $1,06 \mathrm{e}-2$ & $4,15 \mathrm{e}-2$ & $9,00 \mathrm{e}-2$ & 0,153 & 0,315 & 0,512 \\
\hline$f_{4}$ & $7,95 \mathrm{e}-4$ & $5,10 \mathrm{e}-3$ & $2,07 \mathrm{e}-2$ & $4,56 e-2$ & $7,85 \mathrm{e}-2$ & 0,164 & 0,271 \\
\hline$f_{5}$ & $5,7 e-4$ & $3,60 \mathrm{e}-3$ & $1,43 \mathrm{e}-2$ & $3,20 \mathrm{e}-2$ & $5,67 \mathrm{e}-2$ & 0,125 & 0,217 \\
\hline$f_{6}$ & $8,0 \mathrm{e}-4$ & $5,10 \mathrm{e}-3$ & $2,04 \mathrm{e}-2$ & $4,53 e-2$ & $7,90 \mathrm{e}-2$ & 0,170 & 0,286 \\
\hline$f_{10}$ & $5,8 \mathrm{e}-4$ & $3,70 \mathrm{e}-3$ & $1,50 \mathrm{e}-2$ & $3,33 e-2$ & $5,82 \mathrm{e}-2$ & 0,126 & 0,215 \\
\hline$f_{11}$ & $2,3 e-4$ & $6,90 \mathrm{e}-3$ & $1,85 \mathrm{e}-2$ & $3-54 e-2$ & $5,81 \mathrm{e}-2$ & 0,121 & 0,205 \\
\hline \multicolumn{8}{|c|}{ ICAyi } \\
\hline$f 3$ & 99,98 & 99,89 & 99,51 & 98,91 & 98,08 & 95,80 & 92,69 \\
\hline $\mathrm{f} 4$ & 99,99 & 99,94 & 99,76 & 99,45 & 99,02 & 97,82 & 96,14 \\
\hline$f_{5}$ & 99,99 & 99,96 & 99,83 & 99,61 & 99,29 & 98,33 & 96,90 \\
\hline$f_{6}$ & 99,99 & 99,94 & 99,76 & 99,45 & 99,01 & 97,74 & 95,91 \\
\hline$f_{10}$ & 99,99 & 99,96 & 99,82 & 99,60 & 99,27 & 98,32 & 96,94 \\
\hline$f_{11}$ & 99,99 & 99,92 & 99,78 & 99,57 & 99,27 & 98,39 & 97,07 \\
\hline
\end{tabular}


Cuadro 4. Estimación de las ganancias en el límite de la estabilidad.

\begin{tabular}{|c|c|c|c|c|c|c|c|}
\hline \multicolumn{8}{|c|}{ Kcu } \\
\hline$o^{t_{m}}$ & 0,10 & 0,25 & 0,50 & 0,75 & 1,0 & 1,5 & 2,0 \\
\hline$f_{0}$ & 16,34 & 6,93 & 3,81 & 2,78 & 2,26 & 1,76 & 1,52 \\
\hline \multicolumn{8}{|c|}{ Kcui } \\
\hline$f_{3}$ & 21,00 & 9,00 & 5,00 & 3,67 & 3,00 & 2,33 & 2,00 \\
\hline$f_{4}$ & 15,23 & 6,46 & 3,55 & 2,59 & 2,12 & 1,67 & 1,45 \\
\hline$f_{5}$ & 16,49 & 7,00 & 3,85 & 2,81 & 2,29 & 1,79 & 1,54 \\
\hline$f_{6}$ & 17,28 & 7,35 & 4,04 & 2,95 & 2,40 & 1,87 & 1,61 \\
\hline$f_{10}$ & 15,90 & 6,75 & 3,71 & 2,71 & 2,21 & 1,73 & 1,50 \\
\hline$f_{11}$ & 16,27 & 6,90 & 3,78 & 2,76 & 2,25 & 1,75 & 1,52 \\
\hline \multicolumn{8}{|c|}{ \% EKcui } \\
\hline$f_{3}$ & 28,50 & 29,87 & 31,20 & 31,90 & 32,70 & 32,40 & 21,60 \\
\hline$f_{4}$ & $-6,80$ & $-6,84$ & $-6,85$ & $-6,70$ & $-6,10$ & $-5,28$ & $-4,67$ \\
\hline$f_{5}$ & 0,92 & 1,00 & 1,00 & 0,94 & 1,35 & 1,40 & 1,38 \\
\hline$f_{6}$ & 5,75 & 6,00 & 6,10 & 5,97 & 6,32 & 6,01 & 5,60 \\
\hline$f_{10}$ & $-2,70$ & $-2,70$ & $-2,70$ & $-2,70$ & $-2,20$ & $-1,80$ & $-1,51$ \\
\hline$f_{11}$ & $-0,43$ & $-0,49$ & $-0,68$ & $-0,83$ & $-0,49$ & $-0,34$ & $-0,33$ \\
\hline
\end{tabular}

\subsection{Respuesta de frecuencia}

La función tiempo muerto en frecuencia está dada por

$f_{O}(j \omega)=e^{-j \omega t_{m}}$

cuya magnitud es unitaria para todas las frecuencias y su fase - $t_{m}$, esto es, tiende a cero para baja frecuencia y decrece linealmente con la frecuencia, teniendo un valor de $-180^{\circ}$ cuando $t_{m}$.

Una característica importante que debe tener entonces la aproximación del tiempo muerto, es que no afecte el diagrama de Bode de magnitud de la función de transferencia de lazo abierto, esto es, que tenga magnitud unitaria para todas las frecuencias. Esta característica la presentan las aproximaciones mediante el cociente de dos series de Taylor $\left(f_{3}, f_{4}\right)$, las de Padé $\left(f_{3}, f_{5}\right)$ y los cocientes de polos múltiples $\left(f_{3}, f_{6}\right)$. Otras aproximaciones que presentan esta característica son la de Marshall $\left(f_{9}\right)$, Piche $\left(f_{4}, f_{6}\right)$, Gradshteyn y Ryzhik $\left(f_{10}\right)$, y Sthal y Hippe $\left(f_{11}\right)$.

Sin embargo, todas estas aproximaciones tienen limitaciones en cuanto al desfase máximo

que pueden proveer. Mientras que la fase del tiempo muerto puro decrece linealmente con la frecuencia, las aproximaciones anteriores, todas funciones de transferencia de fase no mínima, proveen un desfase máximo de $-180^{\circ}\left(f_{3}\right) \mathrm{o}-360^{\circ}$ $\left(f_{4}, f_{5}, f_{6}, f_{9}, f_{10}, f_{11}\right)$ a alta frecuencia, por lo que serán de preferencia las de mayor grado para estudios en el dominio de la frecuencia.

\section{OBTENCIÓN DE NUEVAS APROXIMACIONES}

Las pruebas comparativas realizadas, demostraron que la aproximación de la función exponencial por medio de las series de Taylor de orden bajo no era adecuada y debían buscarse expresiones polinomiales de primer y segundo orden mejores; que la aproximación de Padé de primer orden, aunque muy utilizada, mostraba índices de calidad inferiores a los obtenidos con cualesquiera de las aproximaciones de segundo orden, debiendo entonces verificarse si era posible encontrar una mejor aproximación de primer orden; y que los mejores resultados se obtuvieron con la aproximación Sthal y Hippe, por lo que se deseó verificar si era posible encontrar alguna otra que lograra superarla. 


\subsection{Aproximaciones de la función exponencial}

Utilizando la función lsqcurvefit de MATLAB ${ }^{\circledR}$ se encontraron expresiones polinomiales para la función $e^{-x}$ en el intervalo $0 \leq x \leq 2,0$, obteniéndose las siguientes aproximaciones para el tiempo muerto

$e^{-t_{m} s} \approx f_{1 n}(s)=1-0,5272 t_{m} s$

$e^{-t_{m} s} \approx f_{2 n}(s)=1-0,8610 t_{m} s+0,2225 t_{m}^{2} s^{2}$

cuyos índices de calidad de la aproximación exponencial (ICAei) fueron 79,21\% y 97,12\%, respectivamente, los que contrastan ampliamente con los índices de la serie de Taylor de primer orden $(0 \%)$ y de segundo orden $(45,79 \%)$.

Es evidente que de requerirse utilizar una aproximación polinomial para el tiempo muerto, las nuevas expresiones $f_{1 n}$ (27) y $f_{2 n}$ (28) brindarán mejores resultados que las series de Taylor.

\subsection{Aproximaciones mediante funciones de transferencia propias}

En una forma similar a la anterior, se obtuvieron aproximaciones de la función exponencial como cocientes de polinomios del tipo (1-ax+bx $\left.x^{2}\right)$ $\left(1+a x+b x^{2}\right)$, determinándose las siguientes nuevas aproximaciones para el tiempo muerto

$e^{-t_{m} s} \approx f_{3 n}(s)=\frac{1-0,4362 t_{m} s}{1+0,4362 t_{m} s}$

$e^{-t_{m} s} \approx f_{4 n}(s)=\frac{1-0,4986 t_{m} s+0,0783 t_{m}^{2} s^{2}}{1+0,4986 t_{m} s+0,0783 t_{m}^{2} s^{2}}$

cuyos índices de calidad de la aproximación exponencial (ICAei) fueron 93,84 \% y 99,90 $\%$ respectivamente, superiores a los índices de las aproximaciones de Padé de primer orden $(89,35 \%)$ y de segundo orden $(99,63 \%)$.
Se probaron también expresiones más generales de la forma $\left(1-a x+b x^{2}\right) /\left(1+c x+d x^{2}\right)$ con una mejora de aproximadamente un $5 \%$ en el índice de calidad para el caso de primer orden, pero despreciable en el de segundo. Como los coeficientes de los polinomios del numerador y del denominador de estas nuevas aproximaciones resultaron ser bastante diferentes entre sí, no se consideraron adecuadas ya que su magnitud no sería unitaria para todas las frecuencias, característica necesaria como se indicó en 3.5.

\subsection{Prueba de las nuevas aproximaciones}

Si bien las nuevas aproximaciones $f_{3 n}$ y $f_{4 n}$ proveen una mejor aproximación de la función exponencial, debió verificarse que su desempeño como representación de un tiempo muerto puro, como parte de la respuesta en el tiempo de un sistema con tiempo muerto y en la determinación de los límites de estabilidad del sistema de control, era también superior al de las aproximaciones existentes.

Como representación de un tiempo muerto puro, sus índices de calidad ICAti fueron 80,96 $\%$ y $86,25 \%$, muy similares a los de las aproximaciones de Padé.

El índice de calidad de la respuesta del sistema de primer orden más tiempo muerto ICAyi promedio de $f_{3 n}$ fue $97,26 \%$ y el de $f_{4 n} 99,09 \%$, ligeramente inferiores a los de las aproximaciones de Padé $(97,84 \%$ y $99,13 \%)$.

Sin embargo en la prueba de estabilidad los resultados fueron ampliamente inferiores. $\mathrm{La}$ aproximación $f_{3 n}$ tuvo un error promedio del $+45,16 \%$ y la $f_{4 n}+2,29 \%$ comparados con el $+29,74 \%$ y $+1,15 \%$ de las aproximaciones de Padé.

El hecho de ofrecer una mejor aproximación de la función exponencial, no garantizó entonces que estas nuevas expresiones tuvieran un mejor desempeño en las pruebas dinámicas. 


\subsection{Optimización de las aproximaciones mediante pruebas dinámicas}

Debido a que los resultados de las comparaciones efectuadas, demostraron que las expresiones obtenidas mediante el ajuste de la función exponencial, no mejoraron el desempeño dinámico de las aproximaciones, se realizó una optimización dinámica de los coeficientes de los polinomios de las aproximaciones utilizando el programa VisSim $^{\mathrm{TM}}$.

Utilizando la misma planta y parámetros utilizados en las pruebas comparativas indicadas en el punto 3.3 anterior, se simuló la planta con el tiempo muerto puro y también con una aproximación de primer orden, y se optimizó el coeficiente de los polinomios del numerador y denominador mediante el método de optimización de Powell, minimizando la diferencia, esto es el error, entre las respuestas al escalón de las dos plantas. Los coeficientes del polinomio óptimo variaron entre 0,4948 y 0,4967 . Utilizando un promedio de los coeficientes encontrados se obtuvo la nueva aproximación óptima de primer orden

$e^{-t_{m} s} \approx f_{3 o p}(s)=\frac{1-0,496 t_{m} s}{1+0,496 t_{m} s}$

la que no difiere significativamente de la aproximación de Padé de primer orden.

Al optimizar el polinomio de segundo orden, se encontró que mientras el parámetro de los términos lineales variaba entre 0,500 y 0,4895 , el de los términos cuadráticos variaba entre $8,974 \cdot 10^{-2}$ y $9,2785 \cdot 10^{-2}$, por lo que se optó por fijar el parámetro del término lineal de los polinomios, en el valor encontrado con la aproximación de primer orden y optimizar solamente el del término cuadrático. La nueva aproximación óptima de segundo orden resultante fue

$e^{-t_{m} s} \approx f_{4 o p}(s)=\frac{1-0,496 t_{m} s+0,091 t_{m}^{2} s^{2}}{1+0,496 t_{m} s+0,091 t_{m}^{2} s^{2}}$

bastante similar a la aproximación de Sthal y Hippe $f_{11}$.
En el caso de la nueva aproximación óptima de primer orden $f_{3 o p}$, como era de esperarse, esta dio exactamente los mismos resultados en las pruebas de respuesta al escalón y de ganancia en el límite de la estabilidad que la aproximación de Padé de primer orden; sin embargo, en la aproximación de la función exponencial dio un índice de calidad del 90,03\%, ligeramente superior al 89,35 \% de la aproximación de Padé.

Las pruebas con la aproximación óptima de segundo orden $f_{4 o p}$, dieron como resultado un índice de calidad de la aproximación de la respuesta al escalón ICAyi del 99,16\% comparable con el 99,13\% obtenido por la aproximación de Padé de segundo orden y el 99,14 \% de la aproximación de Sthal y Hippe.

En cuanto a las pruebas de estabilidad, la aproximación óptima de segundo orden dio un excelente resultado, con un error promedio de sólo el $-0,099 \%$ y valores extremos para $o{ }^{1}$ del $-0,40 \%$ y $+0,20 \%$ para $o$.

En cuanto a la aproximación de la función exponencial, el índice de calidad obtenido con la función optimizada fue del $98,002 \%$, superior al 96,46\% de la aproximación de Sthal y Hippe, pero inferior al 99,63\% de la de Padé de segundo orden.

\section{CONCLUSIONES}

De las pruebas comparativas realizadas se puede concluir que:

1. Las nuevas aproximaciones polinomiales de la función exponencial obtenidas $f_{3 n}$ y $f_{4 n}$, son ampliamente superiores a las aproximaciones por series de Taylor del mismo orden.

2. La nueva aproximación del tiempo muerto, mediante una función de transferencia propia de fase no mínima de primer orden $f_{3 o p}$, provee resultados similares o mejores que la aproximación de Padé de primer orden. 
3. La nueva aproximación mediante una función de transferencia de segundo orden $f_{4 o p}$, provee resultados mejores que la aproximación de Padé de segundo orden y que la de Sthal y Hippe y se puede considerar como mejor en su comportamiento general que todas las otras aproximaciones existentes comparadas.

Se recomienda entonces la utilización de las nuevas aproximaciones del tiempo muerto encontradas en este estudio y en especial el de la función $f_{4 o p}$, en los estudios de los sistemas de control.

\section{APÉNDICE}

Para la evaluación de la bondad de las aproximaciones del tiempo muerto comparadas, se establecieron las siguientes mediciones y criterios:

- Aproximación de la función exponencial

Se definió la Integral de la función Tiempo Muerto (ITMe) como

ITMe $=\int_{O}^{x_{f}} f_{O}(x) d x$

y la Integral del Error Absoluto de la Aproximación (IEAei) de cada aproximación de la función exponencial $f_{i}$ como

IEA $i=\int_{O}^{x_{f}}\left|f_{O}(x)-f_{i}(x)\right| d x$

La calidad de cada aproximación fi estará dada por el Índice de Calidad de la Aproximación (ICAei) definido como

ICAe $=100\left[1-\frac{\text { IEAei }}{\text { ITMe }}\right]$

- Aproximación del tiempo muerto puro

Para comparar cuán bien representaban las aproximaciones la respuesta de un tiempo muerto puro, se definió la Integral de la respuesta del Tiempo Muerto (ITMt)
$I T M t=\int_{0}^{m t_{m}} f_{O}(t) d t=(m-1) t_{m}$

la Integral del Error Absoluto de la Aproximación del tiempo muerto (IEATt)

$I E A t i=\int_{0}^{m t_{m}}\left|f_{o}(t)-f_{i}(t)\right| d t$

y el Índice de Calidad de la Aproximación del tiempo mиerto (ICAti)

$I C A t i=100\left[1-\frac{I E A t i}{I T M t}\right]$

donde $m t_{m}$ es el tiempo necesario para garantizar que la respuesta de todas las funciones hubieran alcanzado su valor final.

- Efecto sobre la respuesta al escalón

En forma similar se definió la Integral de la respuesta al escalón de la planta con tiempo muerto (ITMy)

ITMy $=\int_{0}^{t} y_{o}\left(f_{o}, t\right) d t$

la Integral del Error Absoluto de la Aproximación de la respuesta al escalón (IEAyi) para cada función $f_{i}$

IEAyi $=\int_{0}^{t}\left|y_{o}\left(f_{o}, t\right)-y_{i}\left(f_{i}, t\right)\right| d t$

y el Índice de Calidad de la Aproximación de la respuesta al escalón (ICAyi)

$I C A y i=100\left[1-\frac{I E A y i}{I T M y}\right]$

en donde $y_{O}\left(f_{o}, t\right)$ es la respuesta de la planta con tiempo muerto a una entrada escalón unitario y $y_{i}\left(f_{i}, t\right)$ las respuestas de la planta utilizando las aproximaciones del tiempo muerto.

\section{SIMBOLOGÍA}

$G_{p^{(}}(s) \quad$ Función de transferencia de la planta.

$G_{c}(s) \quad$ Función de transferencia del controlador.

$k_{p} \quad$ Ganancia estática. 


$\begin{array}{ll} & \text { Constante de tiempo. } \\ t_{m} & \text { Tiempo muerto aparente. } \\ { }_{s} & \text { Variable compleja. }\end{array}$

\section{BIBLIOGRAFÍA}

Bogere, M. N. \& Özgen, C. (1989). On-Line controller tuning of second order dead time processes, Chem. Eng. Res. Des., (67), November.

Dwight, H. B. (1961) Tables of integrals and other mathematical data, New York The Macmillan Co.

Gradshteyn, I. S. \& Ryzhik, I. M. (1980). Tables of integrals, series and products. Corrected and enlarged edition. (Ed. A. Jeffrey), Academic Press, Inc.

INRIA (Institut National de Recherche en Informatique et en Automatique). (2003). Scilab 2.7. Extraído de: http://www.scilab. org.

Jutan, A. \& Rodríguez, E. S. (1984). Extension of a new method for on-line controller tuning. The Canadian Journal of Chemical Engineering, (62), Dember.

Kochenburger, R. J. (1972). Computer simulation of dynamic systems, Prentice-Hall, Inc.

Marshall, J. E. (1990). Control of time-delay systems. IEE Control Engineering, Peter Peregrinus Ltd., Series 10.

Martin, J., Smith, C. L., Corripio, A. B. (1975). Controller tuning from simple process models. Instrumentation Technology, 12 (22), November.

O’Dwyer, A. (1996 August). The estimation and compensation of process with time delays. PhD Thesis, School of Electronic
Engineering, Dublin City University, Dublin. Irlanda.

Piche, R. (1990). Low-order rational all pass ap-proximations to e-s. En: Proceedings of the 11th IFAC World Congress on Automatic Control, 2, (139-137). Tallin, Estonia.

Rivera, D. E., Morari H. \& Skogestads. (1986) Internal model control 4: PID controller design. Ind. Eng. Chem. Process Des. Dev., 45, 2173-2182.

Stahl, W. Z. \& Hippe, P. (1987). Design of polo placing controllers for stable and unstable systems with pure time delay. International Journal of Control, 45, 2173 - 2182.

The Mathworks, Inc., (2000) MATLAB 6.5. Extraído de: http://www.matworks.com.

The Mathworks, Inc., (2002), Simulink 5.0, Extraído de: http://www.matworks.com.

Visual Solutions, Inc. (1988). EUA, VisSim 3.0, Extraído de: http://www.vissim.com.

Yuwana, M. \& Seborg, D. E. (1982). A new method for on-line controllers tuning, AIChE Journal, 3 (28), May.

\section{SOBRE EL AUTOR}

\section{Víctor M. Alfaro Ruiz}

Profesor Asociado, Departamento de Automática, Escuela de Ingeniería Eléctrica, Universidad de Costa Rica.

Apartado postal 2-10, 2060 UCR, San José, Costa Rica.

Teléfono: 207-4472, Facsímil. 207-4139

Correo electrónico: valfaro@eie.ucr.ac.cr

El presente trabajo fue realizado como parte del proyecto No 322-A3-007 inscrito en la Vicerrectoría de Investigación de la Universidad de Costa Rica. 